\title{
Estimation of Delta Winding Current and Its Application to a Compensated-Current-Differential Relay for a Y- $\Delta$ Transformer
}

\author{
Yong-Cheol Kang*, Byung-Eun Lee ${ }^{\dagger}$ and En-Shu Jin**
}

\begin{abstract}
The compensated-current-differential relay uses the same restraining current as a conventional relay, but the differential current is modified to compensate for the effects of the exciting current. Delta winding current is necessary to obtain the modified differential current for a Y $-\Delta$ transformer. This paper describes an estimation algorithm of the delta winding current and its application to a compensated-current-differential relay for a Y- $\Delta$ transformer. Prior to saturation, the core-loss current is calculated and used to modify the differential current. When the core first enters saturation, the initial value of the core flux is obtained by inserting the modified differential current into the magnetization curve. This flux value is used to derive the magnetizing current and consequently the modified differential current. The operating performance of the proposed relay was compared against a conventional current differential relay with harmonic blocking. Test results indicate that the proposed relay remained stable during severe magnetic inrush and over-excitation, and its operating time is significantly faster than a conventional relay. The relay is unaffected by the level of remanent flux and does not require an additional restraining or blocking signal to maintain stability. This paper concludes by implementing the proposed algorithm into a prototype relay based on a digital signal processor.
\end{abstract}

Keywords: Y- $\Delta$ transformer, Delta winding current, Modified differential current

\section{Nomenclature}

\begin{tabular}{c|c}
\hline Symbols & Definition \\
\hline$v_{A}, v_{B}, v_{C}$ & Primary voltages \\
\hline$i_{A}, i_{B}, i_{C}$ & Primary currents \\
\hline$v_{a b}, v_{b c}, v_{c a}$ & Secondary voltages \\
\hline$i_{a}, i_{b}, i_{c}$ & Secondary line currents \\
\hline$i_{a b}, i_{b c}, i_{c a}$ & Secondary winding currents \\
\hline$i_{p a}, i_{p b}, i_{p c}$ & Non-circulating components \\
\hline$i_{\Delta}$ & Circulating component \\
\hline$R_{A}, R_{B}, R_{C}$ & Primary winding resistances \\
\hline$L_{l A}, L_{l B}, L_{l C}$ & Primary leakage inductances \\
\hline$R_{a}, R_{b}, R_{c}$ & Secondary winding resistances \\
\hline$L_{l a}, L_{l b}, L_{l c}$ & Secondary leakage inductances \\
\hline$e_{A}, e_{B}, e_{C}$ & Primary induced voltages \\
\hline$e_{a b}, e_{b c}, e_{c a}$ & Secondary induced voltages \\
\hline$R_{c A}, R_{c B}, R_{c C}$ & Core-loss resistances \\
\hline$L_{m A}, L_{m B}, L_{m C}$ & Magnetizing inductances \\
\hline$i_{e A}, i_{e B}, i_{e C}$ & Exciting currents \\
\hline$i_{c A}, i_{c B}, i_{c C}$ & Core-loss currents \\
\hline$i_{m A}, i_{m B}, i_{m C}$ & Magnetizing currents \\
\hline$N_{1}, N_{2}$ & Number of windings \\
\hline
\end{tabular}

\section{Introduction}

Transformer protection relays must be able to distinguish an internal fault from all other operating conditions. During magnetic inrush or over-excitation, a conventional current

\footnotetext{
$\dagger$ Corresponding Author: Department of Electrical Engineering, Chonbuk National University, Korea. (mpeclab@chonbuk.ac.kr)

* Department of Electrical Engineering, Chonbuk National University, Korea. (yckang@chonbuk.ac.kr)

** Department of Electrical engineering, Chonbuk National University, Korea. (jes222kr@yahoo.co.kr)

Received: February 2, 2010; Accepted: March 16, 2010
}

differential relay may mal-operate due to the increased magnitude of the exciting current. To ensure stability, restraining or blocking signals are used to disable or desensitize the relay. Einvall and Linders [1] proposed a composite restraint function based on the $2^{\text {nd }}$ and $5^{\text {th }}$ harmonics; the technique ensures stability but may delay the operating time on an internal fault.

Phadke and Thorp [2] suggested a flux-restrained current differential relay that calculates the flux using the primary voltage. The technique assumes that during magnetic inrush, the magnetizing current and the flux comply with the magnetization curve of the core; but during an internal fault, the flux is proportional to the magnetizing current with a small slope. The technique assumes the primary current is the magnetizing current. Difficulties arise when magnetic inrush occurs on a loaded transformer or during over-excitation.

Techniques have been reported that rely on the electromagnetic equations of a transformer [3], [4]. The operating speed of this type of relay is very fast, but the primary and secondary voltage and current signals are required.

A conventional current differential relay compares the primary and the secondary winding currents. During magnetic inrush or over-excitation, the relay may mal-operate because the exciting current can be significant but cannot be measured. Kang et al. proposed a compensated-currentdifferential relay for transformer protection [5]. The relay uses the same restraining current as a conventional relay, but derives a modified differential current that compensates for both the core-loss and the magnetizing currents. The technique supports a reduction in the operating time, but for a Y- $\Delta$ transformer, the delta winding current must be measured or calculated. 
Delta winding current can be decomposed into two components, i.e. circulating and non-circulating components. The latter could be estimated directly from the line current but the former could not [3]. For an ultra high voltage transmission system, the delta winding current should be known for reliable protection of a conventional differential relay. For example, for the $765 \mathrm{kV}$ system in Korea, current transformers are mounted inside a transformer to measure the delta winding current. This inevitably increases the size and cost of the transformer.

This paper proposes a compensated-current-differential relay for Y- $\Delta$ transformer protection. To apply the compensated-current-differential relay to Y- $\Delta$ transformer protection, the delta winding current should be known. A method to estimate the circulating component of the delta winding current is also described. This estimate is then used to modify the differential current, using a similar technique as in [5]. The operating performance of the proposed relay and a conventional current differential relay with harmonic blocking were then evaluated under various EMTP simulated scenarios including magnetic inrush, internal faults and over-excitation. This paper concludes by implementing the algorithm into a prototype relay based on a digital signal processor.

\section{Compensated-current-differential Relay for Y- $\Delta$ Transformer Protection}

Fig. 1 shows a three-phase Y- $\Delta$ transformer and its

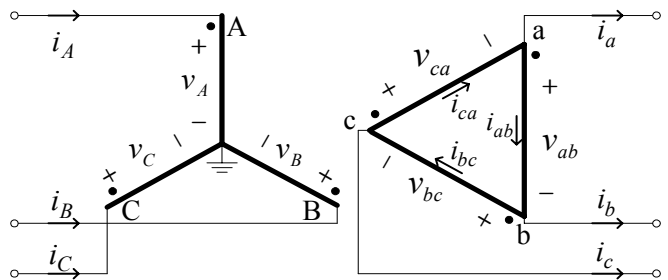

(a) Three phase Y- $\Delta$ transformer

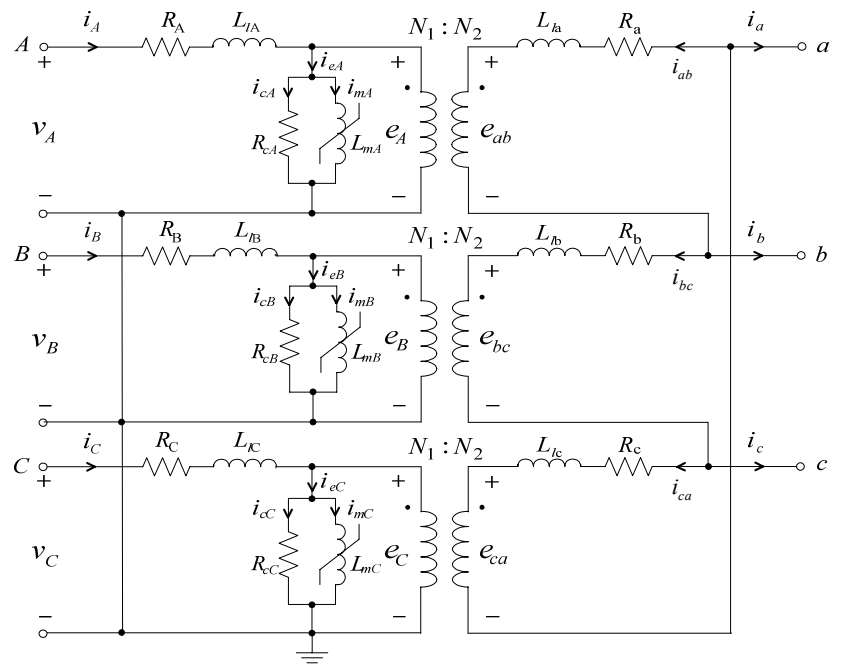

(b) Equivalent circuit

Fig. 1. Three phase Y- $\Delta$ transformer and its equivalent circuit. equivalent circuit. For simplicity, it is assumed that $R_{A} \approx R_{B} \approx R_{C}=R_{1}, L_{l \mathrm{~A}} \approx L_{l B} \approx L_{l C}=L_{l 1}, R_{a} \approx R_{b} \approx R_{c}=R_{2}$, and $L_{l a} \approx L_{l b}$ $\approx L_{l c}=L_{l 2}$; although the derivation is still applicable even if this assumption is not valid.

If there is no internal fault, primary and secondary voltages can be represented by

$$
\begin{gathered}
v_{A}=R_{1} i_{A}+L_{l 1} \frac{d i_{A}}{d t}+e_{A} \\
v_{B}=R_{1} i_{B}+L_{l 1} \frac{d i_{B}}{d t}+e_{B} \\
v_{C}=R_{1} i_{C}+L_{l 1} \frac{d i_{C}}{d t}+e_{C} \\
v_{a b}=R_{2} i_{a b}+L_{l 2} \frac{d i_{a b}}{d t}+e_{a b} \\
v_{b c}=R_{2} i_{b c}+L_{l 2} \frac{d i_{b c}}{d t}+e_{b c} \\
v_{c a}=R_{2} i_{c a}+L_{l 2} \frac{d i_{c a}}{d t}+e_{c a}
\end{gathered}
$$

\subsection{Conventional Current Differential Relay with Harmonic Blocking}

This subsection will describe the main features of a conventional current differential relay with $2^{\text {nd }}$ and $5^{\text {th }}$ harmonic blocking applied to a three-phase Y- $\Delta$ transformer (see logic diagram in Fig. 2). The results obtained with this relay were compared with the proposed relay and are presented in section 4 for the A phase element. The conventional relay derives the magnitude of the fundamental component of the operating differential current $I_{d A}$ using

$$
I_{d A}=\left|\vec{I}_{A}-\vec{I}_{C}-a \vec{I}_{a}\right|
$$

where $a=N_{2} / N_{1}$.

The magnitude of the fundamental component of the restraining current $I_{r A}$ is obtained by

$$
I_{r A}=\frac{\left|\vec{I}_{A}-\vec{I}_{C}+a \vec{I}_{a}\right|}{2}
$$

The characteristic of the differential relay (87R) is given by

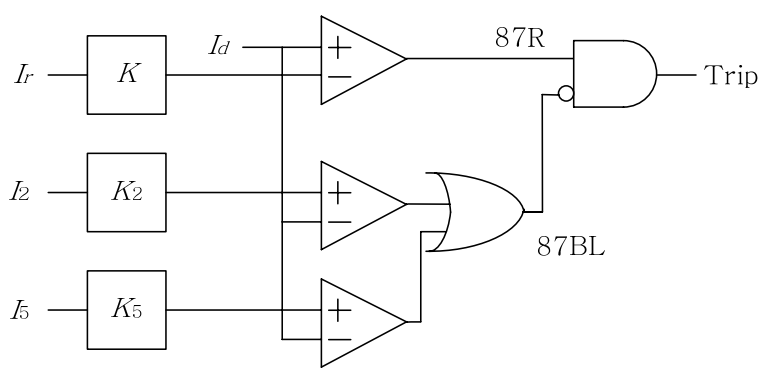

Fig. 2. Logic diagram of a conventional differential relay with harmonic blocking. 


$$
I_{d A} \geq I_{o f f s e t}+K I_{r A}
$$

where $I_{\text {offset }}$ and $K$ were set to $15 \mathrm{~A}$ and 0.3 respectively. A blocking signal (87BL) is activated if either (10) or (11) is satisfied.

$$
\begin{aligned}
& I_{d} \leq K_{2} I_{2} \\
& I_{d} \leq K_{5} I_{2}
\end{aligned}
$$

where $I_{2}$ and $I_{5}$ are the $2^{\text {nd }}$ and $5^{\text {th }}$ harmonic components of the differential current and $K_{2}$ and $K_{5}$ are constants, set at 12 and 15 , respectively. These values were chosen to ensure the trip signal is blocked for all magnetic inrush and over-excitation conditions on the model test system.

\subsection{Compensated-current-differential Relay for Y- $\Delta$ Transformer Protection}

$I_{d A}$ of the conventional relay does not consider the effect of the exciting current; thus, during magnetic inrush or over-excitation, $I_{d A}$ can exceed the operating threshold and the relay may mal-operate. The proposed relay uses the same restraining current as the conventional relay but modifies the differential current to include the effect of the exciting current.

To derive the compensated-current-differential relay for Y- $\Delta$ transformer protection, the delta winding current should be calculated before the differential current is modified. Once the delta winding current is obtained, the procedure of deriving the modified differential current is the same as [5]. To cope with the remanent flux, the relay obtains the modified differential current separately 'before saturation' and 'during saturation'. Before saturation, since the magnetizing current is negligible, only the core-loss current is calculated and used to obtain the modified differential current. During saturation, because the magnetizing current becomes significant, both the core-loss and magnetizing currents are calculated to obtain the modified differential current.

\subsubsection{Estimation of the Delta Winding Current}

Delta winding currents $i_{a b}, i_{b c}$ and $i_{c a}$ can be decomposed into the two components, i.e. the non-circulating component $\left(i_{p a}, i_{p b}\right.$, and $\left.i_{p c}\right)$ and the circulating component $i_{\Delta}$.

$$
i_{a b}=i_{p a}+i_{\Delta}, i_{b c}=i_{p b}+i_{\Delta}, i_{c a}=i_{p c}+i_{\Delta}
$$

In [3], $i_{p a}, i_{p b}$, and $i_{p c}$ were obtained from the line currents using

$$
i_{p a}=\frac{i_{b}-i_{a}}{3}, i_{p b}=\frac{i_{c}-i_{b}}{3}, i_{p c}=\frac{i_{a}-i_{c}}{3}
$$

However, $i_{\Delta}$ was not obtained. This paper estimate $i_{\Delta}$ using the following procedure:
- (1)-(3) are re-arranged to yield

$$
\begin{aligned}
& e_{A}=v_{A}-R_{1} i_{A}-L_{l 1} \frac{d i_{A}}{d t} \\
& e_{B}=v_{B}-R_{1} i_{B}-L_{l 1} \frac{d i_{B}}{d t} \\
& e_{C}=v_{C}-R_{1} i_{C}-L_{l 1} \frac{d i_{C}}{d t}
\end{aligned}
$$

- Kirchhoff's voltage law is then applied to the large loop of the delta winding of Fig. 1 and (12) is used to yield

$$
e_{a b}+e_{b c}+e_{c a}=L_{l 2} \frac{d\left(3 i_{\Delta}\right)}{d t}+R_{2}\left(3 i_{\Delta}\right)
$$

- Left hand side of (17) is obtained from $e_{A}, e_{B}$, and $e_{C}$ by taking into consideration the turns ratio, i.e:

$$
\frac{e_{A}}{e_{a b}}=\frac{e_{B}}{e_{b c}}=\frac{e_{C}}{e_{b c}}=\frac{N_{1}}{N_{2}}
$$

Thus, $i_{\Delta}$ is obtained by solving (17), with the differential term approximated using the backward Euler method. Finally $i_{a b}, i_{b c}$ and $i_{c a}$ are estimated using (12).

Once $i_{a b}, i_{b c}$ and $i_{c a}$ are obtained, the modified differential current is estimated using the same technique as in [5]; for clarity, this is briefly described below.

\subsubsection{Prior to Saturation (sat_ind $=0$ )}

The modified differential current $i_{d A}$ is calculated prior to saturation using

$$
i_{d A}=i_{A}+a i_{a b}-i_{c A}
$$

where, with reference to Fig. $1 \mathrm{~b}, i_{c A}$ can be estimated if $e_{A}$ is derived using (14) and $R_{c A}$ is obtained experimentally. The "prior to saturation" modified differential current is then derived using (19), where

$$
i_{c A}=\frac{e_{A}}{R_{c A}}
$$

\subsubsection{During Saturation (sat_ind $=1$ )}

The modified differential current $i_{d A}$ remains very small until the core enters saturation, the magnetizing current then becomes significant and must be included in the equation used to calculate $i_{d A}$, i.e.:

$$
i_{d A}=i_{A}+a i_{a b}-i_{c A}-i_{m A}
$$

In (21), $i_{c A}$ is calculated using (20) and $i_{m A}$ is estimated using the following procedure:

If $i_{d A}$ in (19) exceeds a threshold, the start of saturation is 
detected and "sat ind $=1$ ". At this instant $i_{d A}$ is regarded as the magnetizing current; hence, the core flux can be obtained by inserting $i_{d A}$ into the magnetization curve (see Fig. 3).

This flux value is considered to be $\lambda_{A 0}$ and hence $\lambda_{A}$ can be calculated using

$$
\lambda_{A}=\int_{t_{0}}^{t} e_{A} d t+\lambda_{A 0}
$$

where $\lambda_{A 0}$ is the core flux at $t=t_{0}$. Therefore, $i_{m A}$ is estimated by inserting $\lambda_{A}$ into the magnetization curve. The technique correctly estimates $i_{m A}$ and is not affected by the level of remanent flux.

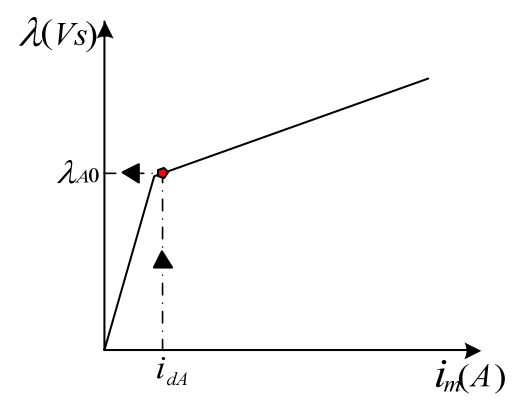

Fig. 3. Estimation of $\lambda_{A 0}$.

\section{Case Studies}

Fig. 4 shows a single line diagram of the simulated system. A two-winding $\mathrm{Y}-\Delta$ transformer $(154 \mathrm{kV} / 13 \mathrm{kV}, 55$ MVA) was modeled using EMTP at a sampling rate of 64 samples/cycle (s/c). The techniques described in [6] were used to represent an internal winding fault.

The hysteresis characteristic of the core was modeled in the EMTP subroutine HYSDAT using a type-96 element, with a saturation point of $(40 \mathrm{~A}, 334 \mathrm{Vs})$. Butterworth $2^{\text {nd }}$ order filters with a stop-band cut-off frequency of $1920 \mathrm{~Hz}$ were used as anti-aliasing filters. The threshold for detecting the start of saturation was set at $80 \mathrm{~A}$.

The proposed relay and a conventional relay with harmonic blocking were evaluated during various operating conditions, including magnetic inrush, internal faults and over-excitation. Results are presented for the A-phase element.

$$
\left.\left.6 \text { [GVA] } \frac{50[\mathrm{~km}]}{\mathrm{Zs}_{\mathrm{s}}}\right\}^{\mathrm{Y}}\right\}^{-\Delta} \begin{array}{cc}
55[\mathrm{MVA}] \\
\mathrm{LOAD}
\end{array}
$$

Fig. 4. Single-line diagram of the model system.

\subsection{Magnetic Inrush}

The magnitude of the inrush current depends on the energization angle, the remanent flux in the core, and the load current.
Case 1: Energization angle of $0 \mathrm{deg}, 80 \%$ remanent flux, no load

Fig. 5 shows the results obtained when the conventional relay was tested using the data from case 1 . The transformer was energized at $29.2 \mathrm{~ms}$ and because the energization angle was zero deep saturation occurred. As shown in Fig. 5a, the conventional differential current $i_{d A}$ consists of a sequence of pulses; the magnitude of the first pulse is $2300 \mathrm{~A}$ and it contains a large $2^{\text {nd }}$ harmonic component; note: the thick solid line, the thin solid line and the dotted lines represent the fundamental, $2^{\text {nd }}$ and $5^{\text {th }}$ component of the differential current, respectively. The differential relay ' $87 \mathrm{R}$ ' enters its operating region at $32.3 \mathrm{~ms}$, but was blocked by ' $87 \mathrm{BL}$ ', this was activated at $29.4 \mathrm{~ms}$ and remains active. Hence, as expected, no trip signal was issued, because $K_{2}$ had been set to block the relay for all magnetic inrush conditions.

Fig. 6 shows how the proposed relay responded to case 1 . Note: $i_{p a}, i_{\Delta}$ and $i_{a b}$ in Fig. 6a are obtained from (13), (17) and (12) respectively and $i_{p a}$ is zero because no load current flows in case 1 . The relay detects the start of saturation at $31.5 \mathrm{~ms}$ and the initial flux corresponds to $342 \mathrm{Vs}$. Eq. (22) is used to calculate $\lambda_{A}$ and $i_{m A}$ is obtained by inserting $\lambda_{A}$ into the magnetization curve. As shown in Fig. 6b, the resulting modified differential current $i_{d A}$ is reduced to a small value (compare Fig. $6 \mathrm{~b}$ and Fig. 5a). Thus, the proposed relay does not enter the operating region whilst the conventional relay does (see Fig. 6c). This is because the proposed relay successfully compensates for the effect of the exciting current.
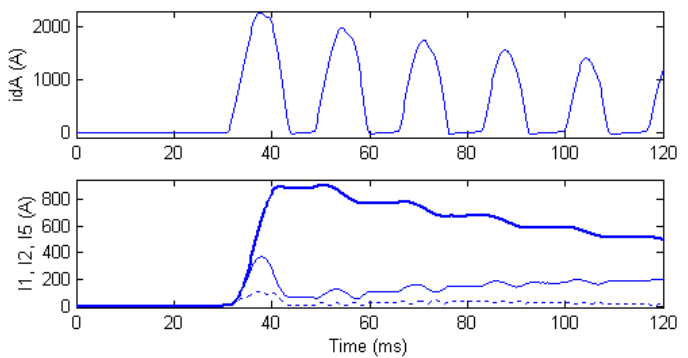

(a) $i_{d A}$ and its harmonic components
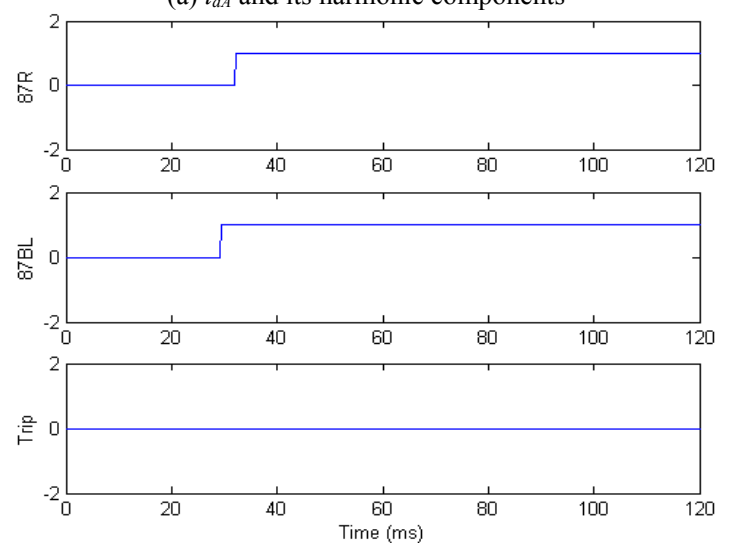

(b) $87 \mathrm{R}, 87 \mathrm{BL}$ and trip signal

Fig. 5. Results of the conventional relay with harmonic blocking (case 1). 

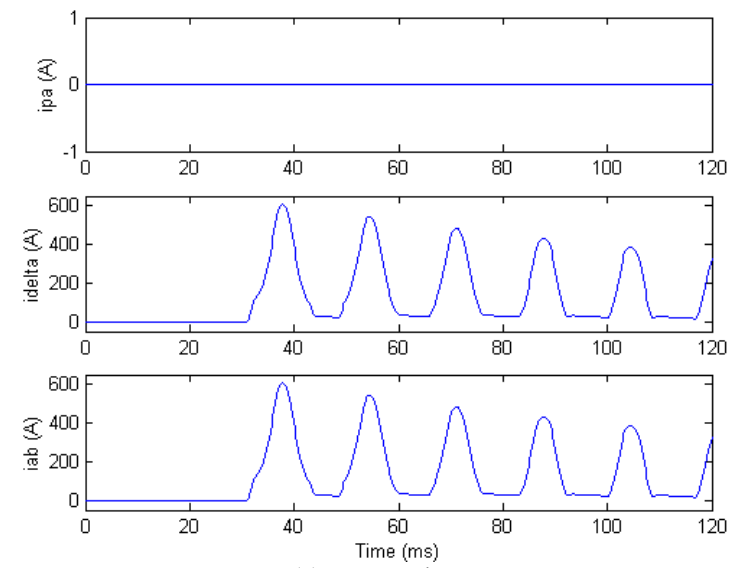

(a) $i_{p a}, i_{\Delta}$ and $i_{a b}$
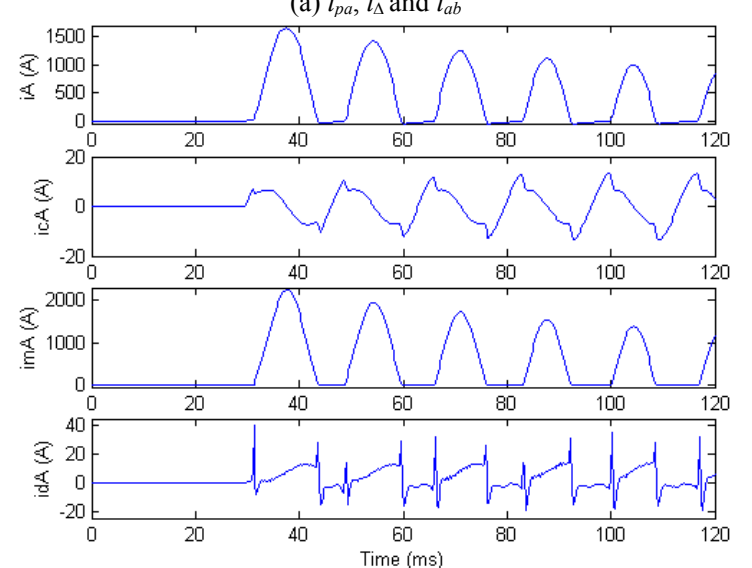

(b) $i_{A}, i_{c A}, i_{m A}$ and $i_{d A}$

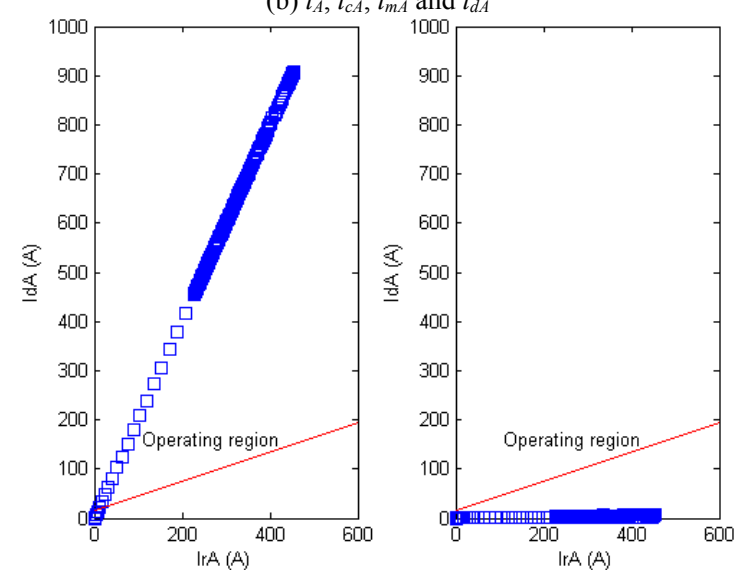

(c) Conventional (left) and proposed (right) relays

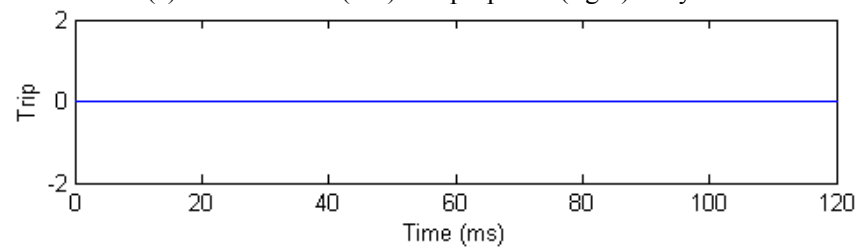

(d) Trip signal

Fig. 6. Results of the proposed relay (case 1).

\subsection{Internal Winding Faults}

Various types of fault were applied to the A-phase of the primary winding and the resulting voltage and current signals used to test the conventional and the proposed relay.
Case 2: Turn-to-ground A-phase fault, located $80 \%$ from the neutral end and 0 deg inception angle

Figs. 7 and 8 show the operating response of the conventional and proposed relay when the fault occurs at $29.2 \mathrm{~ms}$. The conventional relay ' $87 \mathrm{R}$ ' enters the operating region at $32.0 \mathrm{~ms}$ but is blocked by the activation of ' $87 \mathrm{BL}$ ' at 29.7 ms. At $54.2 \mathrm{~ms}$ ' $87 \mathrm{BL}$ ' resets and the relay correctly issues a trip signal.

As with the previous cases, the proposed relay calculates $i_{a b}$, detects the start of saturation at $30.2 \mathrm{~ms}$ and evaluates an initial core flux of 346.0 Vs. However, soon after the start of saturation, $i_{m A}$ decreases to a low value but increases again at $42 \mathrm{~ms}$ (Fig. 8b). This large $i_{m}$ is irrelevant because the core is unlikely to saturate on an internal fault. This is because on an internal fault, $v_{A}$ is significantly reduced whilst $i_{A}$ is significantly increased; hence, the third term at the right hand side of (1) is dominant compared with the first and second terms, i.e.:

$$
e_{A}=v_{A}-R_{A} i_{A}-L_{l A} \frac{d i_{A}(t)}{d t} \approx-L_{l A} \frac{d i_{A}(t)}{d t}=\frac{d \lambda_{A}}{d t}
$$
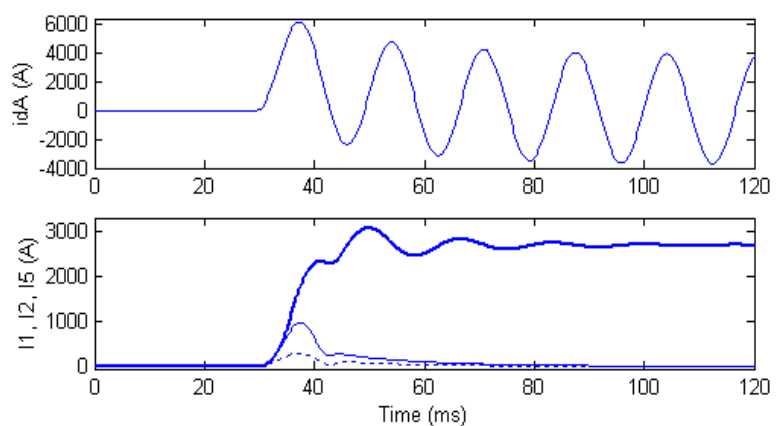

(a) $i_{d A}$ and its harmonic components
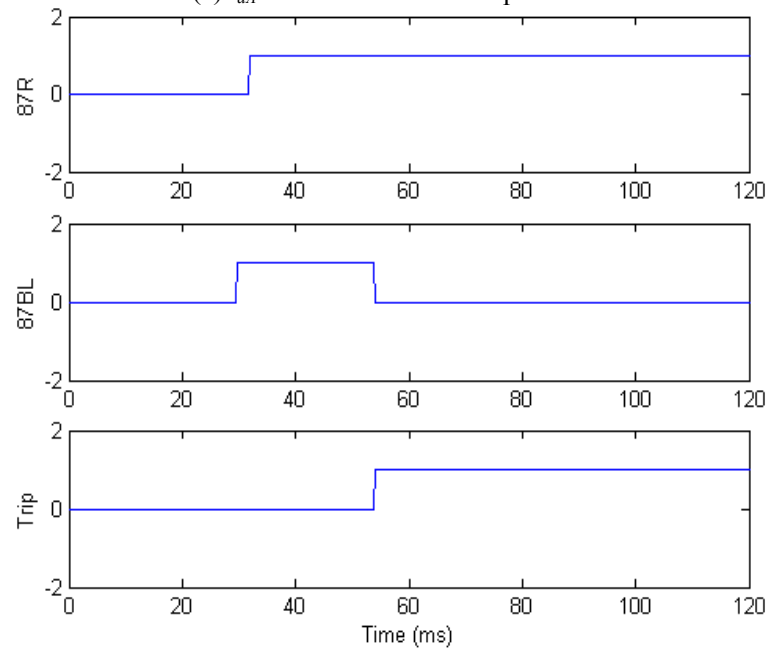

(b) $87 \mathrm{R}, 87 \mathrm{BL}$ and trip signal

Fig. 7. Results of the conventional relay with harmonic blocking (case 2). 

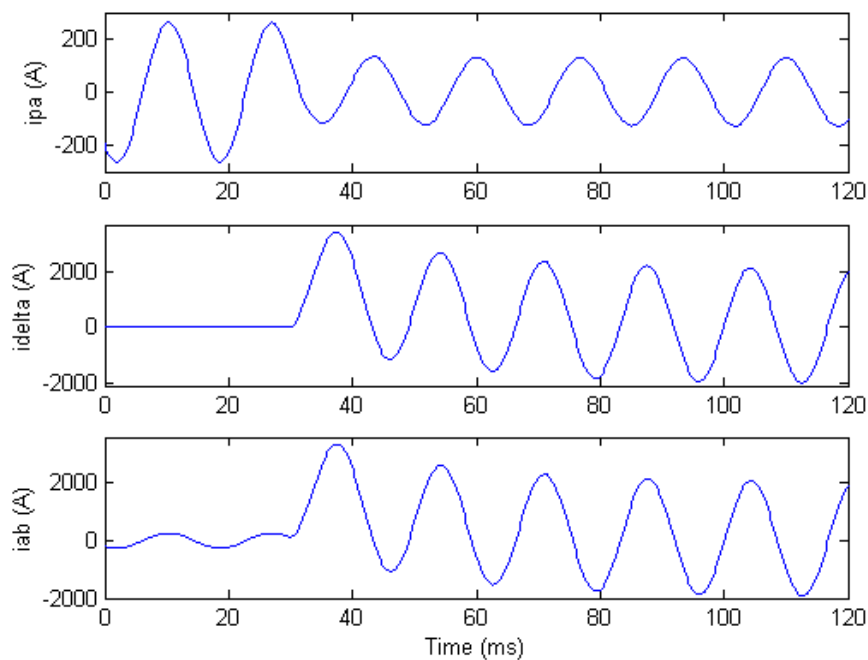

(a) $i_{p a}, i_{\Delta}$ and $i_{a b}$
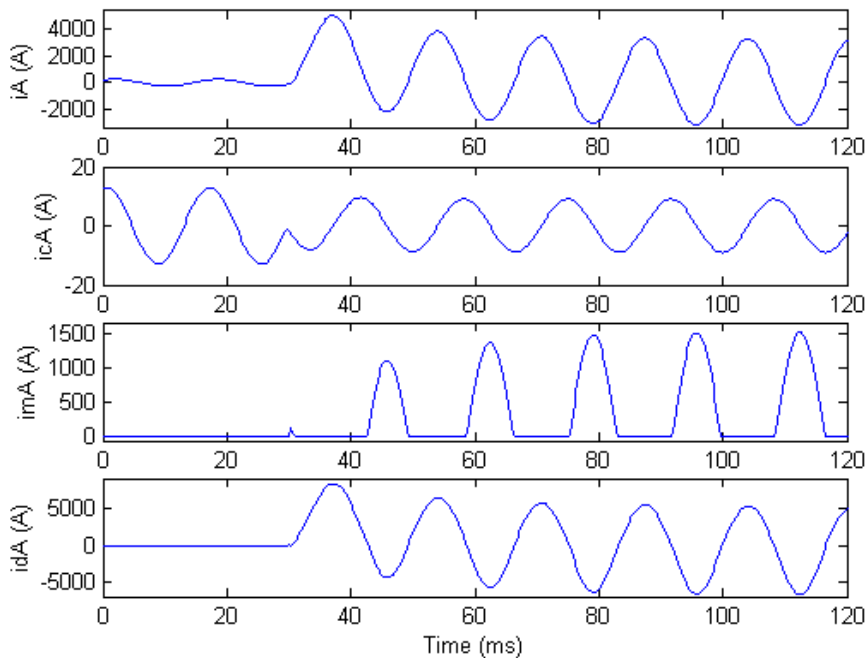

(b) $i_{A}, i_{c A}, i_{m A}$ and $i_{d A}$
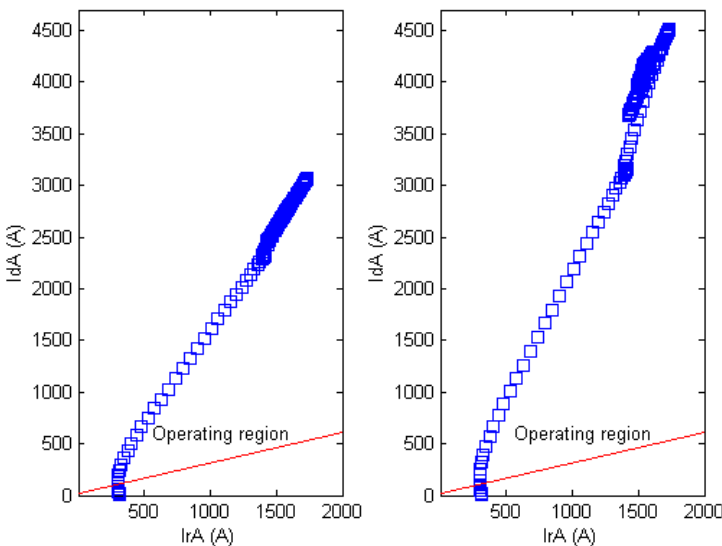

(c) Conventional (left) and proposed (right) relays

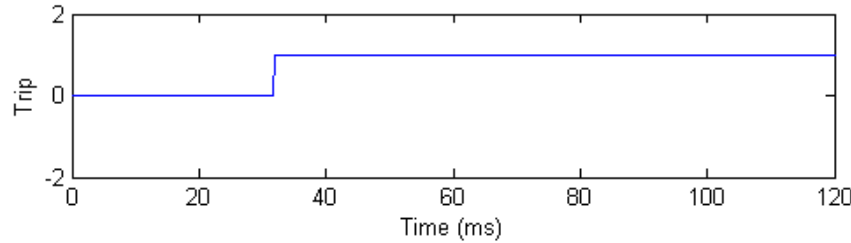

(d) Trip signal

Fig. 8. Results of the proposed relay (case 2).
Thus, the direction of $\lambda_{A}$ is opposite to the direction of $i_{A}$, which is in phase with the conventional $i_{d A}$; consequently, the direction of $i_{m A}$ is opposite to the direction of $i_{d A}$, as can be seen by comparing Figs. $7 \mathrm{a}$ and $8 \mathrm{~b}$. Therefore, the compensated $i_{d A}$ is not reduced by the subtraction of $i_{m A}$. The compensated relay issues a trip signal at $32.0 \mathrm{~ms}$, which is the same operation as ' $87 \mathrm{R}$ ', i.e. $22.2 \mathrm{~ms}$ faster than the conventional relay.

\subsection{Over-excitation}

Case 3: Over-voltage of $150 \%$ applied, full load

Figs. 9 and 10 show the results obtained when the transformer was over-excited by applying $150 \%$ of the rated voltage at $29.2 \mathrm{~ms}$ (case 3 ). In the conventional relay, operation of ' $87 \mathrm{R}$ ' at $38.8 \mathrm{~ms}$, was blocked by activation of ' $87 \mathrm{BL}$ ' at $31.5 \mathrm{~ms}$, and hence no trip signal was issued. The result is as expected, since $K_{5}$ was set to block the trip signal during over-excitation. The proposed relay calculates $i_{a b}$; detects the start of saturation at $36.2 \mathrm{~ms}$ and determines an initial core flux of $350.2 \mathrm{Vs}$. The relay calculates $i_{m A}$ and $i_{c A}$ and uses them to derive $i_{d A}$. The value remains small and consequently the relay does not enter the operating region.
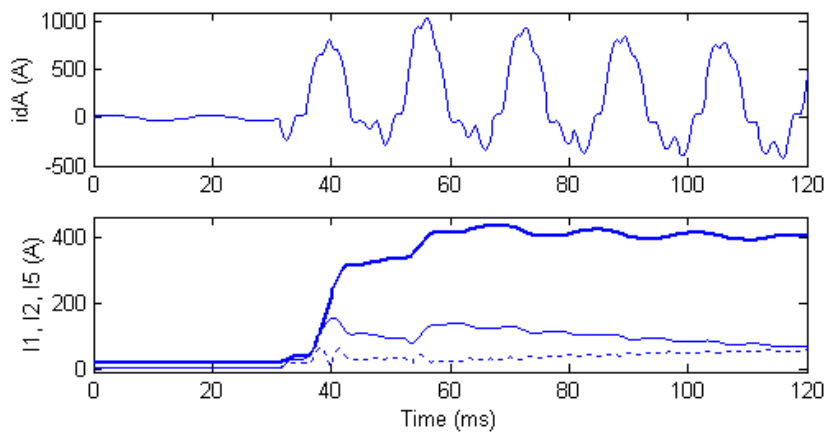

(a) $i_{d A}$ and its harmonic components
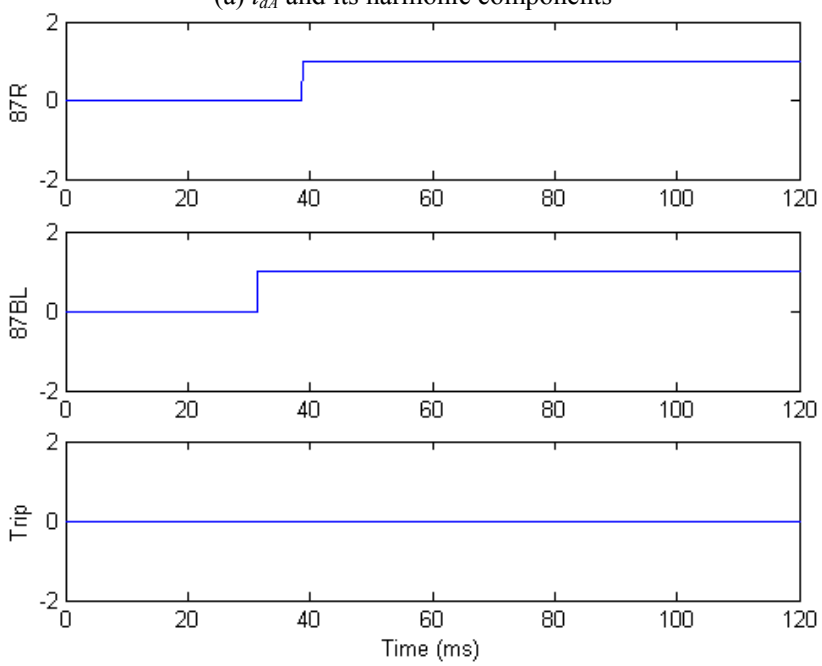

(b) $87 \mathrm{R}, 87 \mathrm{BL}$ and trip signal

Fig. 9. Results of the conventional relay with harmonic blocking (case 3). 

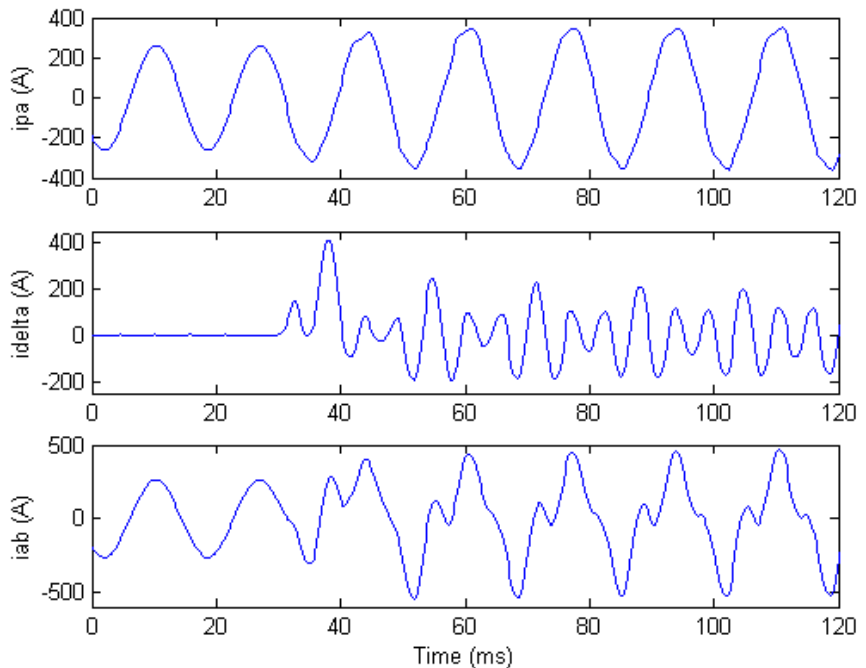

(a) $i_{p a}, i_{\Delta}$ and $i_{a b}$
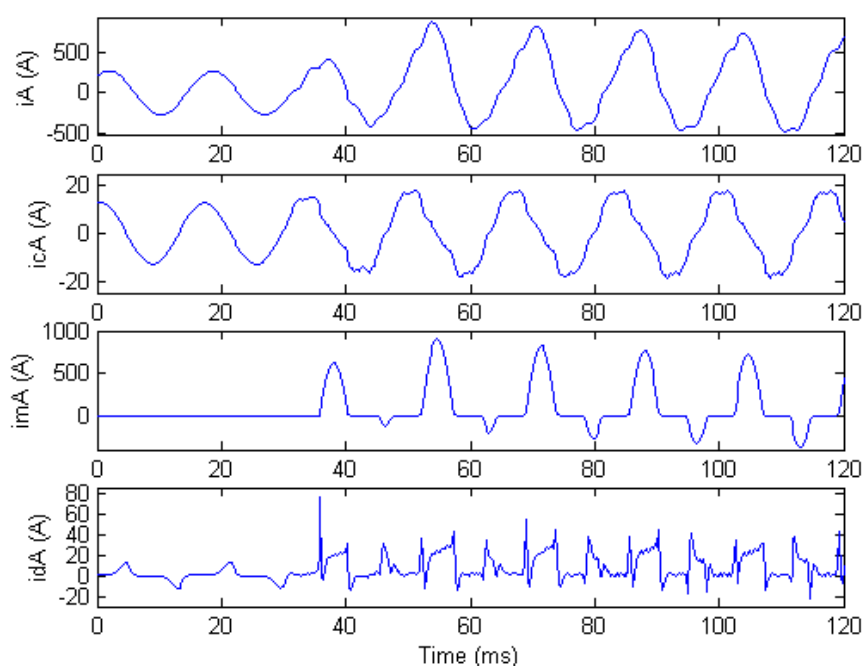

(b) $i_{A}, i_{c A}, i_{m A}$ and $i_{d A}$
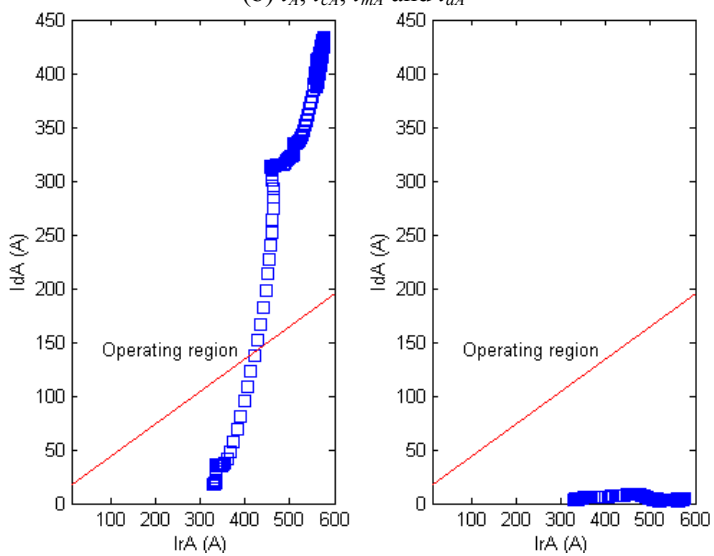

(c) Conventional (left) and proposed (right) relays

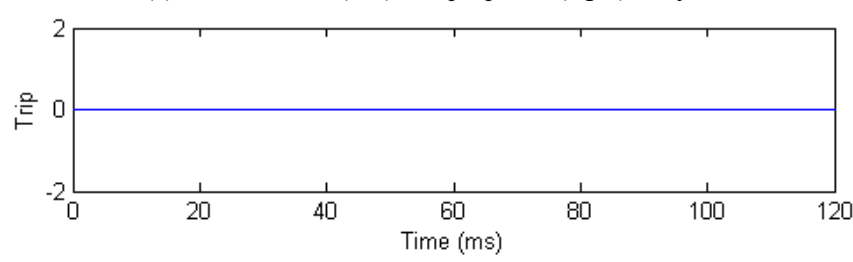

(d) Trip signal

Fig. 10. Results of the proposed relay (case 3).

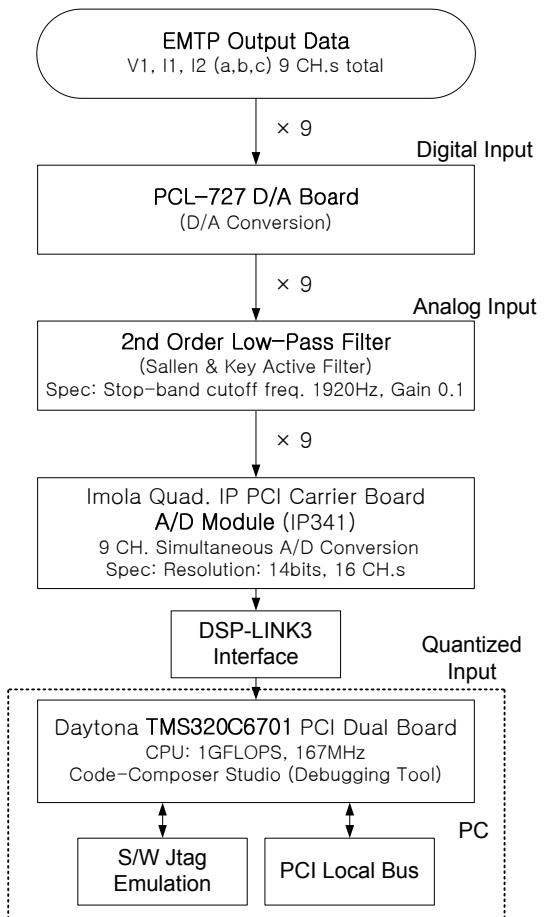

Fig. 11. Configuration of hardware implementation.

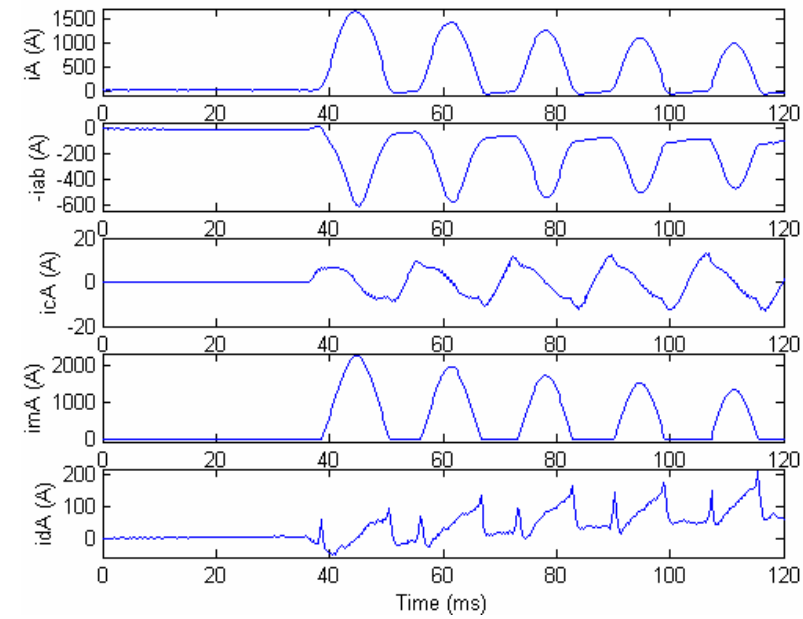

(a) Values $i_{A}, i_{a b}, i_{c A}, i_{m A}$, and $i_{d A}$ for the proposed relay
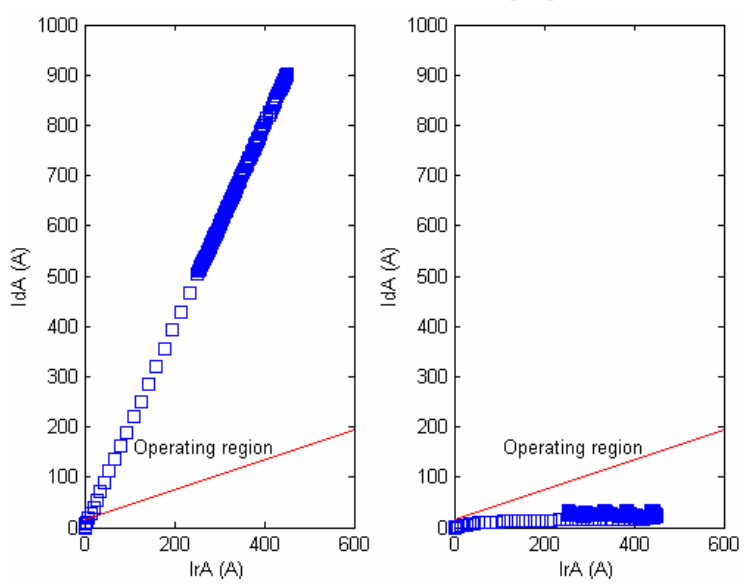

(b) Conventional (left) and proposed (right) relays

Fig. 12. Hardware implementation results from case 1. 


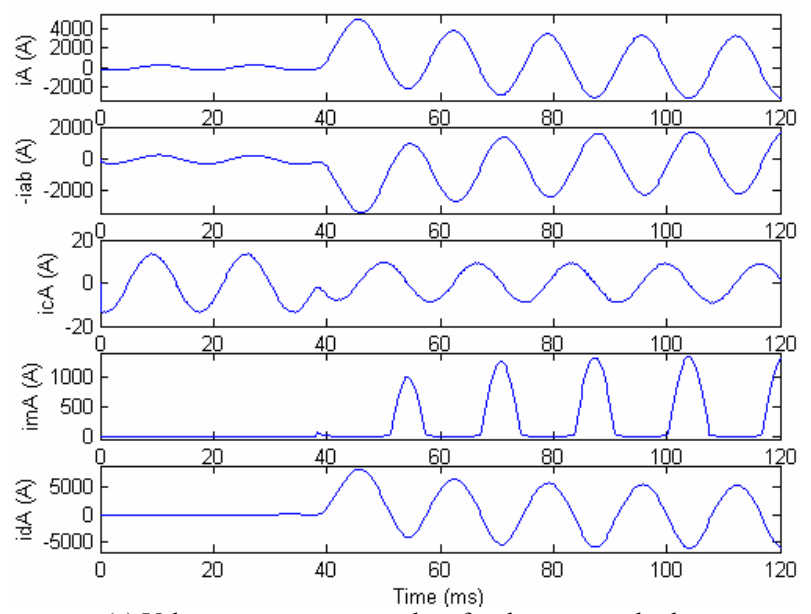

(a) Values $i_{A}, i_{a b}, i_{c A}, i_{m A}$, and $i_{d A}$ for the proposed relay
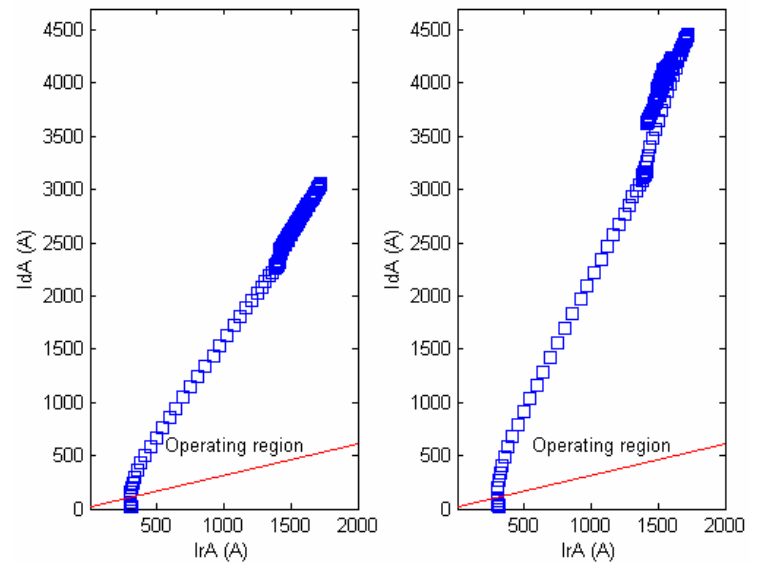

(b) Conventional (left) and proposed (right) relays

Fig. 13. Hardware implementation results from case 2 .

\section{Hardware Implementation Test}

This section shows the results obtained when the compensated algorithm was implemented on a hardware platform based on a TMS320C6701 digital signal processor (see Fig. 11). The three voltages and six currents generated by EMTP are converted into analogue signals using a PCL$727 \mathrm{D} / \mathrm{A}$ board. The signals are then passed through Butterworth $2^{\text {nd }}$ order filters $\left(f_{c}=1920 \mathrm{~Hz}\right)$ to the $14-\mathrm{bit} \mathrm{A} / \mathrm{D}$ converters operating at a sampling rate of $64 \mathrm{~s} / \mathrm{c}$.

Figs. 12 and 13 show the results obtained when the hardware implementation was evaluated using the voltage and current signals described in cases 1 and 2, respectively. The results are very similar to those shown in Figs. 5 and 6, and Figs. 7 and 8 . As expected, the proposed relay remains stable in case 1 and the trip signal is activated in case 2.

\section{Conclusion}

This paper describes a compensated-current-differential relay for the $\mathrm{Y}-\Delta$ transformer and a procedure for estimating the delta winding current. To cope with remanent flux, the modified differential current was determined both 'prior to saturation' and 'during saturation'. For the former, the proposed relay calculates the core-loss current and uses it to obtain the modified differential current. With the latter, the core flux is estimated and used as an initial value to calculate the core flux and the magnetizing current. The relay obtains the modified differential current by subtracting the core-loss current and the magnetizing current from the measured differential current.

The performance of the relay was investigated and compared with a conventional relay that includes harmonic blocking. Both relays were evaluated under various operating conditions, including magnetic inrush, internal faults and over-excitation. The modified differential current remains very small during magnetic inrush and overexcitation and the operating time of the proposed relay is significantly faster than the conventional relay. To investigate the feasibility of building a commercial relay, a prototype relay was constructed using a digital-signalprocessing-based hardware platform and tested using a relay test set. Results described in the paper, show that the relay can successfully discriminate between magnetic inrush and an internal fault.

The relay is highly sensitive and operates correctly at all levels of remanent flux, it also remains stable during magnetic inrush and over-excitation. The relay does not require the inclusion of blocking techniques that often delay the operating time of conventional transformer differential relays.

\section{Acknowledgements}

This work is the outcome of a Manpower Development Program for Energy \& Resources supported by the Ministry of Knowledge and Economy (MKE)

\section{References}

[1] C. H. Einvall and J. R. Linders, "A Three-phase Differential Relay for Transformer Protection," IEEE Trans. on PAS, Vol. 94, No. 6, pp. 1971-1980, Nov./Dec. 1975.

[2] A. G. Phadke and J. S. Thorp, "A New ComputerBased Flux-Restrained Current-Differential Relay for Power Transformer Protection," IEEE Trans. on PAS, Vol. 102, No. 11, pp. 3624-3629, Nov. 1983.

[3] M. S. Sachdev, T. S. Sidhu and H. C. Wood, “A Digital Relaying Algorithm for Detection Transformer Winding Faults," IEEE Trans. on PWRD, Vol. 4, No. 3, pp. 1638-1648, Jul. 1989.

[4] Y. C. Kang and B. E. Lee, "A Transformer Protection Relay Based on Induced Voltages," KIEE Trans on Power Engineering, Vol. 3-A, No. 2, pp.70-78, Jun. 2003.

[5] Y. C. Kang, E. S. Jin, S. H. Kang and P. A. Crossley, "Compensated-current differential relay for protec- 
tion of transformers," IEE Proc. Gener. Trans. Distr., 2004, Vol. 151, No. 4, pp. 548-554.

[6] P. Bastard, P. Bertrand, M. Meunier, "A Transformer Model for Winding Fault Studies," IEEE Trans. on PWRD, Vol. 9, No.2, pp. 690-699, Apr. 1994.

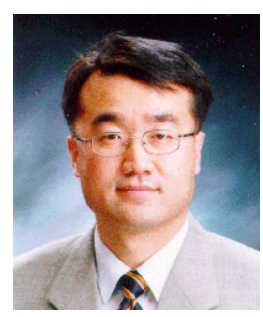

Yong-Cheol Kang received his B.S., M.S. and Ph.D. degrees from Seoul National University, Korea, in 1991, 1993, and 1997, respectively. $\mathrm{He}$ is now a professor at Chonbuk National University, Korea. His research interest is the development of new protection systems for power systems using digital signal processing techniques.

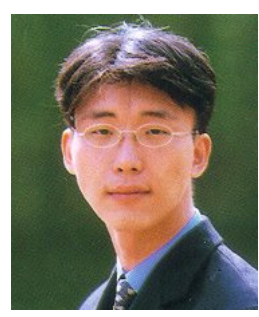

Byung-Eun Lee received his B.S. and M.S. degrees from Chonbuk National University, Korea, in 1999 and 2001, respectively.

He is currently studying for his Ph.D. degree at Chonbuk National University. His research interest is power system protection.

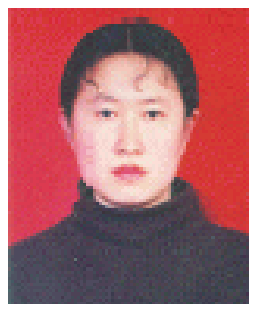

En-Shu Jin received her B.S. and M.S. degrees from the Northeast China Institute of Electric Power Engineering, China, in 1994 and 1999, respectively. She received her Ph.D. degree from Chonbuk National University, Korea, in 2005. Her research interest is power system protection. 